\title{
Fuzzy Model Predictive Controller for Artificial Pancreas
}

\author{
M. El Hachimi, A. Ballouk, A. Baghdad \\ Laboratory of Electronics, Energy, Automatic \& Data Processing (LEEA\&TI) FST- Mohammedia University Hassan II \\ of Casablanca, Morocco
}

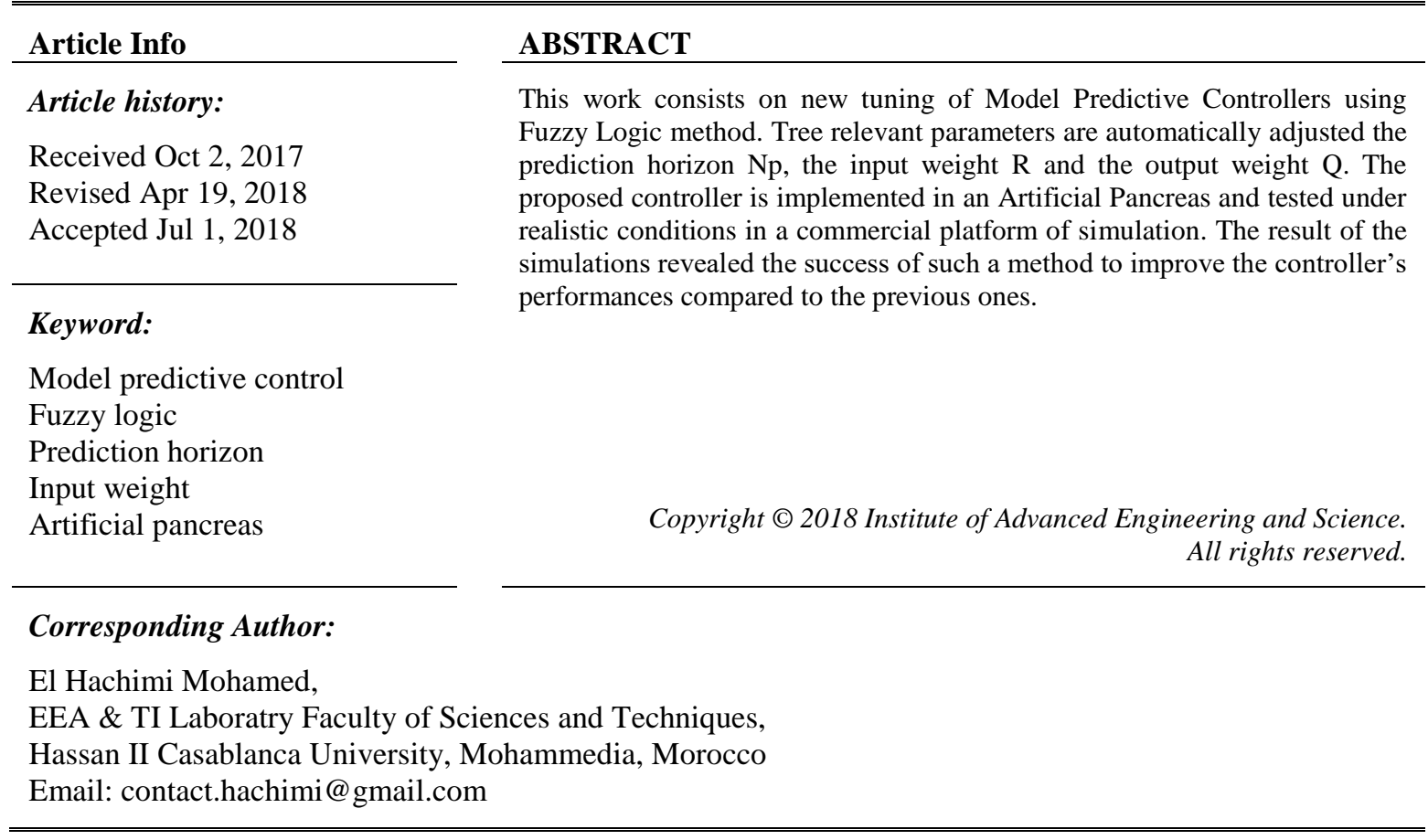

\section{INTRODUCTION}

Model predictive control (MPC) is one of the most successful techniques of advanced control in industry due to its explicit consideration of hard constraint in the optimization, the optimal character of its solution and its capacity to predict future evolution of the system [1]. Until now, just a few guidelines related to MPC parameters tuning exist in literatures. Indeed, these parameters are frequently determined via trial and error procedure. This parameters can be input and output weights in the objective function, prediction and control horizons and constraints. Instead of using an auto-tuning which still a limited technique, it is more interesting to apply an external optimization algorithm. An appropriate automatic tuning of MPC parameters can remarkably ameliorate the performance of control. Many works dealing with automatic tuning of MPC are available in the literature. The paper of Garigga presents a general review of different tuning methods [2]. Trierweiler and Farinab [3] developed an algorithm to tune MPC based on the system degree of directionality and the attainable performance, but the method is complex and requires special competences for implementation and an easier adjustment method is required. Ali et al. [4] applied the concept of fuzzy logic to optimize the parameters of the predictive control. They measure the performance violations and establish the fuzzy rules which constitute the general regulation directives available in the literature for determining the new values of the parameters. Vega et al. [5] presents a nominal model of the process to solve a mixed sensitivity problem with constraints using a frequency domain methods. Despite the significant progress realized in this domain, the development of a general method is still a challenge due the difficulty to perform analytical studies and complexity of methods. The purpose of this paper is to design a control algorithm that can overcomes actual challenges in the automatic glucose control. The principle of the new method is to introduce a new formulation of the cost function of MPC that gives a fast controller capable to reject rapidly the effect of meal intake and avoid hypoglycemia. An automatic tuning of Model Predictive Control using Fuzzy Logic algorithm is presented. The proposed tuning adjust online tree parameters of MPC to ameliorate control performances. 


\section{RESEARCH METHOD}

\subsection{Model}

The proposed tuning is implemented in an Artificial Pancreas (AP) witch is one of the most challenging control problems. Difficult dynamics, interaction between process variables, and the presence of disturbances contribute to the complexity of the blood glucose control, AP is an automated system that manages blood glucose and reduce T1DM risks like, hypo/hyperglycemia, and it mimics the glucose regulating function of a healthy pancreas. AP Device System consists of three components: A continuous glucose monitoring system (CGM), an insulin infusion pump and a control algorithm that connect the CGM and the insulin infusion pump, it determines and injects the right amount of insulin over time. Figure 1 shows components of the AP.

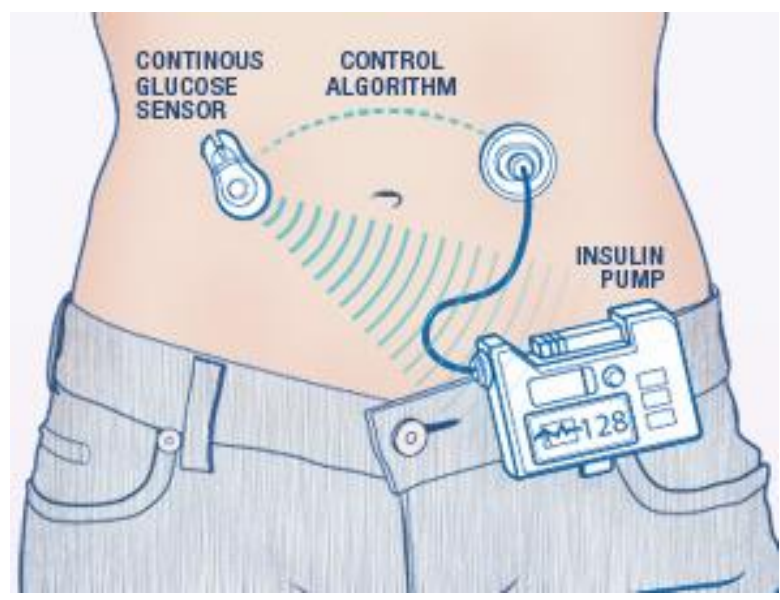

Figure 1 Components of an Artificial Pancreas

The model used in this study [6] is linearized around a steady-state that has been reached by applying the basal rate specific to the subject $\mathrm{U}_{\mathrm{BASAL}} \mathrm{U} / \mathrm{h}$, and lead to a steady-state output ys $=110 \mathrm{mg} / \mathrm{dL}$, the transfer function of the system is:

$$
\frac{y\left(z^{-1}\right)}{u\left(z^{-1}\right)}=\frac{1800 F c}{u_{T D I}} \cdot \frac{z^{-3}}{\left(1-p_{1} Z^{-1}\right)\left(1-p_{2} Z^{-1}\right)^{2}}
$$

With $\mathrm{U}\left(\mathrm{Z}^{-1}\right)$ and $\mathrm{Y}\left(\mathrm{Z}^{-1}\right)$ are the $\mathrm{Z}$-transform of the time-domain signals of the input $\mathrm{Ui}$ and the output $\mathrm{Y} i$, respectively. $\mathrm{Z}^{-1}$ is the backwards shift operator, $\mathrm{c}:=-60\left(1-\mathrm{p}_{1}\right)\left(1-\mathrm{p}_{2}\right)^{2}$ is a constant employed to set the correct gain, to covert the unit, $\mathrm{F}:=1.5$ (unitless ) is a safety factor, $\mathrm{p}_{1}=0.98, \mathrm{p}_{2}=0.965$ are poles of the transfer function and $U_{T D I}[U]$ is the subject specific total daily insulin amount.

\subsection{Controller Design}

Model Predictive Control (MPC) is a class of computer control algorithms that solves an online optimization problem at each sampling point. The concept of MPC is shown in Figure 2. MPC utilizes predictive outputs $y_{k}$ which is estimated within $N p$ steps (prediction horizon) in the future using an internal model. It minimize an objective function, which consists of weighted sum of tracking error of predictive outputs $y_{k}$ to set points $y_{r e f}$, manipulated signals $u_{k}$, penalized with the input weight $\mathrm{R}$ and the output weight Q. MPC problem can be formulated as an optimization problem, which determine input signals $\boldsymbol{u}(k)$, ..., $\boldsymbol{u}(k+N u-1)$ within $\mathbf{N u}$ steps (control horizon) in the future so that the objective function is minimized considering constraints in [7]. 


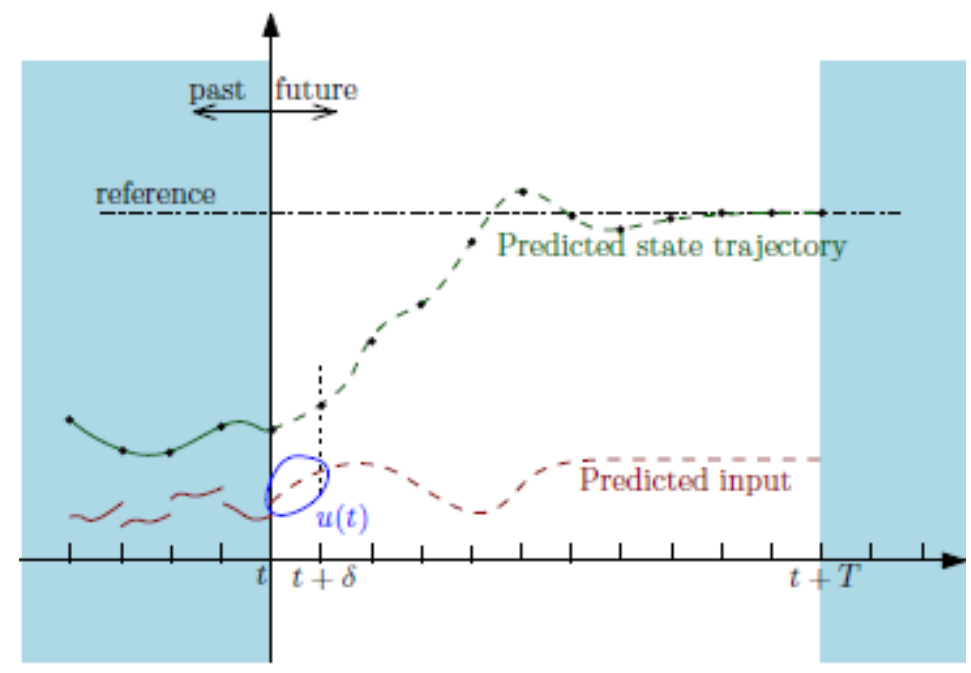

Figure 2. Model Predictive Control Process

The formulation of the optimization problem is:

$$
\left\{u_{0}^{*}, \ldots, u_{N u-1}^{*}\right\}:=\arg \min _{\left\{u_{0}, \ldots, u N_{u-1}\right\}} J\left(x_{i},\left\{u_{0}, \ldots, u_{N_{u-1}}\right\}\right)
$$

With cost function:

$$
J(.):=\sum_{k=1}^{N p(k)} Q(k)\left(y_{k}-y_{r e f}\right)^{2}+\sum_{k=0}^{N_{u-1}} R(k) u_{k}^{2}
$$

\subsection{MPC Tuning method}

\subsubsection{Fuzzy logic concept}

Fuzzy systems allow to prevail a relative truth as membership function that takes values between 0 and 1. The concept of fuzzy theory was introduced by Zadeh (1965). FL can handle uncertainties, imprecision and incomplete data. Indeed it can model non-linear systems and complex functions [8]. A Fuzzy Logic System (FLS) is generally composed of three parts: fuzzification, inference engine and defuzzification. - The fuzzification part corresponds to the definition of linguistic variables of inputs and outputs. - The inference part corresponds to the definition of rules describing the system working. $\bullet$ The defuzzification part computes outputs command. The block diagram of an FLC is shown in Figure 3.

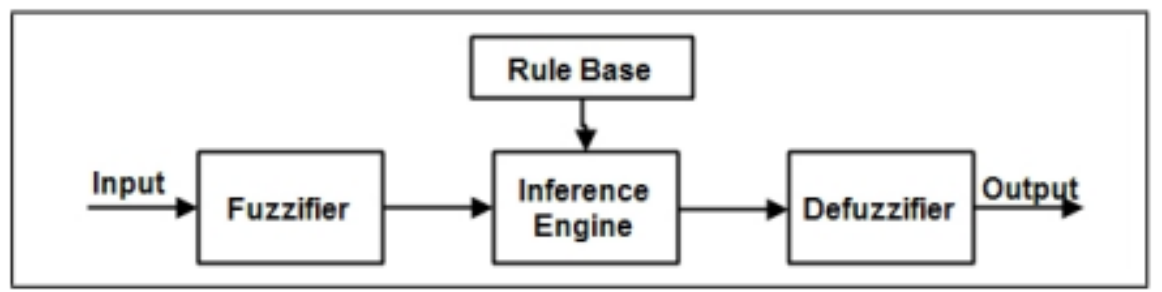

Figure 3. Architecture of fuzzy controller

\subsubsection{Synthesis of the fuzzy controller}

A new tuning technique is presented in this section. This method adapts online the parameters of MPC in order to ameliorate closed loop performance by a rapid rejection of disturbance and avoid overshoot causing hypoglycemia and big peak with long duration of hyperglycemia. In general the desired 
performances in glycemic control are maintaining proper speed of the response, reducing overshoot and rejecting disturbances. Two different input fuzzy sets and one output fuzzy set are used. Figure 4 shows the first input set. We define the scaled bound violation if lower bound is violated as:

$B=\frac{y_{j}^{l}(k+m)-y_{j}(k+m)}{y_{j}^{l}(k+m)}$

If upper bound is violated as:

$A=\frac{y_{i}(k+m)-y_{j}^{u}(k+m)}{y_{j}^{u}(k+m)}$

Equations above define $A$ and $B$ as negative value if the corresponding bound is not violated and positive otherwise.

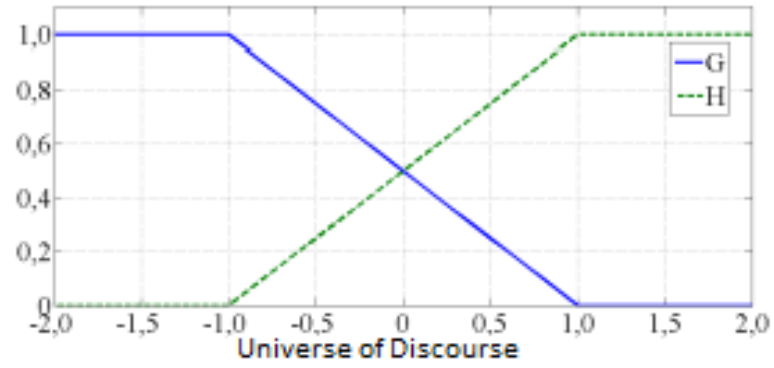

Figure 4. Fuzzy set for bound violation

Equation 6 is related to both bound violation the upper and the lower one. With $Y_{1}$ and $Y_{u}$ are the lower and upper bound, $Y_{j}$ is the predicted output, $\mathrm{j}$ is the iteration index, $m$ is the index of maximum violation for the output and $k$ is the sampling time.

$C=\frac{y_{j}(k+m)-y_{j}(k+m-1)}{y_{j}(k+m)}$

Figure 5 shows the second input fuzzy set. The set is composed of three functions called positive $(\mathrm{P})$, zero $(\mathrm{Z})$ and Negative $(\mathrm{N})$, these functions are denoted by $\mu_{P}, \mu_{Z}$ and $\mu_{N}$, respectively. The scaled violation rate is the second input to the fuzzy, it is defined as:

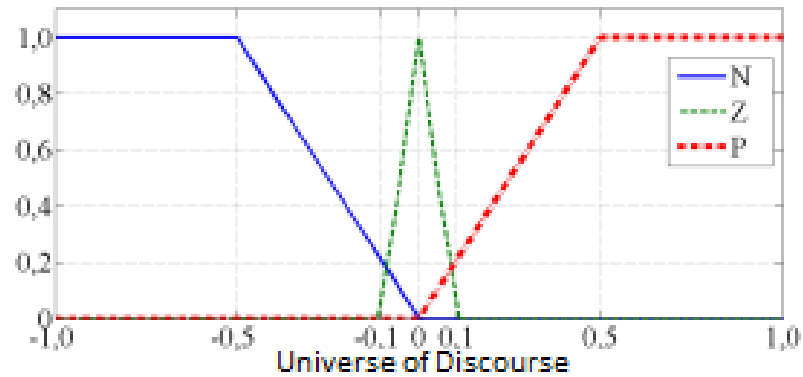

Figure 5. Fuzzy set for bound violation rate 
Figure 6 shows the output fuzzy sets for $R, Q$ and Np symbolized by LN, SN, Z, SP and LP. The membership functions $\mu_{5}, \mu_{4}, \mu_{3}, \mu_{2}$, and $\mu_{1}$ represent respectively these sets. The general understanding of the role of $R, Q$ and $\mathrm{Np}$ on the closed-loop is given by the rules in Table 1.

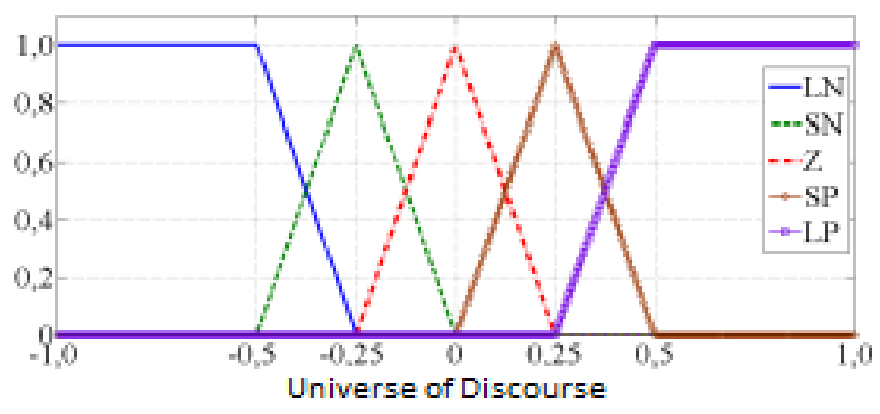

Figure 6. Output fuzzy set

Table 1 shows the basic rules governing the tuning guidelines. $\mu_{P}, \mu_{\lambda}$ and $\mu_{\gamma}$ are the rules output for $\mathrm{R}, \mathrm{Q}$ and $\mathrm{Np}$ respectively.

Table 1. The Base Rules

\begin{tabular}{|c|c|c|c|c|}
\hline No. & Rule & Result of $R$ & Result of $Q$ & Result of $\mathrm{Np}$ \\
\hline $\mathrm{R} 1$ & If $A$ is $H$ and $B$ is $H$ & Then $\mu_{\lambda}$ is SN & Then $\mu_{\gamma}$ is SP & Then $\mu_{P}$ is SP \\
\hline $\mathrm{R} 2$ & If $A$ is $G$ and $B$ is $G$ & Then $\mu_{\lambda}$ is LN & Then $\mu_{\gamma}$ is SP & Then $\mu_{P}$ is SP \\
\hline $\mathrm{R} 3$ & If $A$ is $G$ and $B$ is $H$ & Then $\mu_{\lambda}$ is $\mathrm{Z}$ & Then $\mu_{\gamma}$ is $\mathrm{Z}$ & Then $\mu_{P}$ is $\mathrm{Z}$ \\
\hline $\mathrm{R} 4$ & If $A$ is $H$ and $C$ is $G$ & Then $\mu_{\lambda}$ is $\mathrm{Z}$ & Then $\mu_{\gamma}$ is $\mathrm{Z}$ & Then $\mu_{P}$ is $\mathrm{Z}$ \\
\hline R5 & If $A$ is $H$ and $C$ is $P$ & Then $\mu_{\lambda}$ is $\mathrm{SN}$ & Then $\mu_{\gamma}$ is SP & Then $\mu_{P}$ is SP \\
\hline R6 & If $A$ is $H$ and $C$ is $Z$ & Then $\mu_{\lambda}$ is SP & Then $\mu_{\gamma}$ is SN & Then $\mu_{P}$ is $\mathrm{SN}$ \\
\hline $\mathrm{R} 7$ & If $A$ is $H$ and $C$ is $N$ & Then $\mu_{\lambda}$ is $\mathrm{Z}$ & Then $\mu_{\gamma}$ is Z & Then $\mu_{P}$ is $\mathrm{Z}$ \\
\hline $\mathrm{R} 8$ & If $B$ is $G$ and $C$ is $P$ & Then $\mu_{\lambda}$ is $\mathrm{SN}$ & Then $\mu_{\gamma}$ is SP & Then $\mu_{P}$ is SP \\
\hline R9 & If $B$ is $G$ and $C$ is $Z$ & Then $\mu_{\lambda}$ is SP & Then $\mu_{\gamma}$ is $\mathrm{SN}$ & Then $\mu_{P}$ is $\mathrm{SN}$ \\
\hline $\mathrm{R} 10$ & If $B$ is $G$ and $C$ is $N$ & Then $\mu_{\lambda}$ is Z & Then $\mu_{\gamma}$ is Z & Then $\mu_{P}$ is $\mathrm{Z}$ \\
\hline
\end{tabular}

After transforming the rule into mathematical expressions, the center of area (COA) method can be applied to find the MPC parameters R and Q as follows:

$W(Z)=\frac{\sum_{j=1}^{n_{R}} \sum_{i=1}^{n_{f}} \mu_{j, i}(z) \delta_{i}}{\sum_{j=1}^{n_{R}} \sum_{i=1}^{n_{f}} \mu_{j, i}(z)}$

The argument $Z$ can represent $\mathrm{R}$ or $\mathrm{Q}$, for the output of the prediction horizon $\mathrm{Np}$ is determined as follows:

$W(N p)=\sum_{j=1}^{n_{R}} \sum_{i=1}^{n_{f}} \mu_{j, i}(N p) \delta_{i}$

Where $n_{f}$ is the number of output membership functions, $n_{R}$ is the number of rules equals to 10 in this paper and $\delta i$ is value for the location of the center of $\mu_{\mathrm{i}} . i$ is fixed and pre-assigned, the MPC parameters can be changed by at the most $\pm 50 \%$, therefore the arbitrary values for $\delta$ are selected taking in consideration this variation.

Then set MPC parameters as:

$$
\begin{aligned}
& R(k)=R(k)\left(1+\frac{W_{k}(R) g_{i}}{g_{m}}\right), i=1, \ldots, N u \\
& Q(k)=Q(k)\left(1+W_{k}(Q)\right)
\end{aligned}
$$


$\mathrm{Np}=\mathrm{Np}+W_{k}(N p)$

\subsubsection{FZ-MPC Algorithm Flowchart}

FZ-MPC algorithm flowchart is described below:

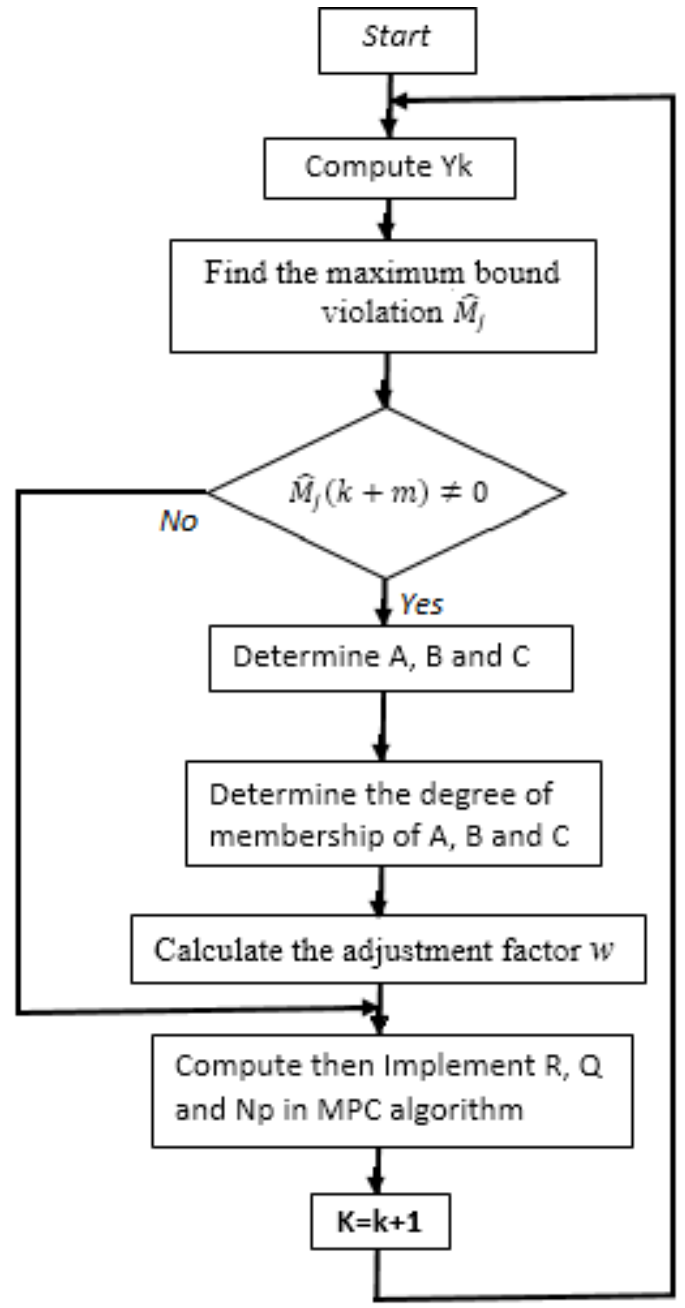

\section{RESULTS AND DISCUSSION}

The FZ-MPC algorithm was tested under the UVa/Padova simulator in order to evaluate their performances. The simulator was approved by the U.S. Food and Drug Administration (FDA) [9] for verification of control algorithms for AP before doing clinical trials on T1DM subjects, this trials are equivalent to animal test. A comparison to non adapted MPC used in [10] is done under a realistic scenario, this controller uses a fixe and a manual tuning of the controller with $\mathrm{R}=500, \mathrm{Q}=0.9, \mathrm{~Np}=8$ and $\mathrm{Nu}=5$ while the FZ-MPC parameters are in the intervals $14 \leq R \leq 7000,0 \leq Q \leq 1,3 \leq N p \leq 10$.

The simulator contains a set of different virtual patients with varying parameters, such as weight, age, gender, 30 in silico subjects of the commercial version (v3) of the UVa/Padova simulator are used in this study to evaluate the control performance of the FL tuning of MPC, this subjects are composed from 10 adults, 10 children and 10 adolescents. Simulations started at 6:00 pm and took 28 hours to be concluded. Every simulation included three meals during the day: Dinner 60 gram carbohydrate ( $\mathrm{gCHO}$ ), breakfast 60 ( $\mathrm{gCHO}$ ), lungh 70 ( $\mathrm{gCHO}$ ) meals consumed at 8:00PM, 07:00AM, and 1:00PM, respectively.

Figure 7 depicts the mean of the blood-glucose and insulin delivery trajectories for the 30 subjects during time of simulations under a realistic scenario. From the resulting plot it can be seen that the evolution of glycemia after the meal intake reaches values near to $400 \mathrm{mg} / \mathrm{dl}$ in the case of non-adapted MPC, however it doesn't exceed the $300 \mathrm{mg} / \mathrm{dl}$ for the proposed tuning. Referring to Figures 7 we can confirm that the duration of hyperglycemia in the case of FZ-MPC is shorter, this will eliminate long term complication of diabetes. 


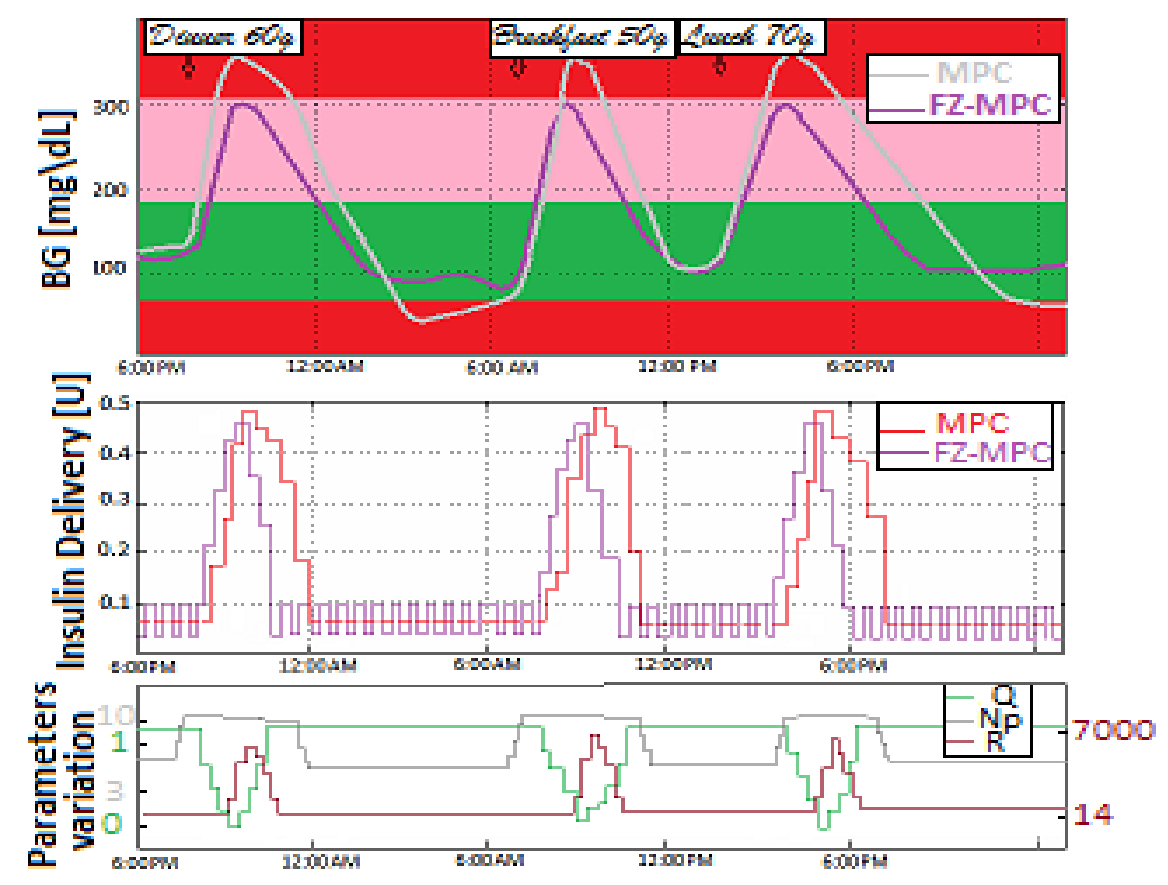

Figure 7. Simulation progress [h]: Mean glucose (A) and Insulin delivery (B) for 30 In-silico subjects controlled by sMPC and aMPC

The proposed method success in reducing the amount of insulin injected comparing to non-adaptive controller this due to the acceleration of command action. Furthermore the post-prandial peak of glycemia is dropped compared to the classical controller. The secret behind the success of the fuzzy tuning is the intelligent management of decisive MPC parameters. This one manages to reduce the value of $\mathrm{R}$ greatly in order to accelerate the tracking of reference without inducing overshoots. For the second parameter the automatic tuning tends to increase the value of at the beginning of change in blood glucose. Then, it is minimized to make the response more conservative thus avoiding hypoglycemia and taking into consideration the delay in insulin absorption. The horizon of prediction is extended when the glycemia change to predict precisely the future evolution and it is reduced elsewhere to reduce computational time.

To conclude discussion of results, performance of the finding method has been clearly demonstrated. The proposed method can overcome limit of the compared algorithm. The non-adapted approach fail to maintain blood glucose in a safe range due to delay in rejection, in addition to some hypoglycemia related to excess in insulin injection. The proposed controller achieves good performance by making the response conservative when the system is stable and accelerating control when a change in blood glucose level happens.

\section{CONCLUSION}

In this paper, we presented new tuning of MPC using fuzzy logic method. Tree MPC parameters are automatically adjusted the prediction horizon $\mathrm{Np}$, the input weight $\mathrm{R}$ and the output weight $\mathrm{Q}$. The attractive feature of the proposed tuning algorithm is the minimal computation time required because it requires no solution of optimization problems or complex mathematical analysis. The simulation of the FZ-MPC applied to Artificial Pancreas demonstrates the capability of the tuning algorithm to ameliorate performance of the closed loop by rapid rejection of disturbance and an avoidance of overshoot causing hypoglycemia and big peak of hyperglycemia. 


\section{ACKNOWLEDGEMENTS}

This work returns the framework of the research project SISA1 "Mini intelligent Power plant" began between research center SISA and our University. We are anxious to think the Hassan II University of Casablanca for the financing of this project.

\section{REFERENCES}

[1] S.J.Qin and T.A.Badgwell, "A survey of industrial model predictive control technology", Control Engineering Practice, No.11, pp733-764, 2003.

[2] Garriga, J.L. and Soroush, M. "Model predictive control tuning methods: a review. Industrial \& Engineering Chemistry Research" 49(8), 3505-3515, 2010.

[3] Trierweiler, J.O., Farinab, L.A. "RPN tuning strategy for model predictive control" J. of Process Control, 13(7), 591-598, 2003.

[4] E. Ali, A. Al-Ghazzawi "On-line Tuning of Model Predictive Controllers Using Fuzzy Logic" The Canadian Journal of Chemical Engineering, Volume 81, October 2003.

[5] Vega, P., Francisco, M. and Sanz, E., Norm "based approaches for automatic tuning of Model Based Predictive Control" In Proceedings of European Congress of Chemical Engineering ECCE-6 (2007).

[6] Lee, J., Dassau, E., Gondhalekar, R., Seborg, D. E., Pinsker, J. E. and Doyle III, F. J. (2016) 'Personalized MPC Strategy with a Dynamic IOB Algorithm and Enhanced, Asymmetric Cost Function for Superior Automated Glucose Control', Industrial and Engineering Chemistry Research, Under Review.

[7] J. Lee, Ravi Gondhalekar, E. Dassau, F. J. Doyle III, "Shaping the MPC Cost Function for Superior Automated Glucose Control”IND ENG CHEM RES Volume 49, Issue 7, 2016, Pages 779-784.

[8] S. Baisa B. Purwahyudi K. Kuspijani "Control Strategy for PWM Voltage Source Converter Using Fuzzy Logic for Adjustable Speed DC Motor" International Journal of Power Electronics and Drive System (IJPEDS) Vol. 8, No. 1, March 2017, pp. 51 58 ISSN: 2088-8694, DOI: 10.11591/ijpeds.v8i1.pp51-58.

[9] Kovatchev BP, Cobelli C, Renard E, et al. "Multinational study of subcutaneous model-predictive closed-loop control in type 1 diabetes mellitus: summary of the results" J Diabetes Sci Technol 2010;4:1374-1381. 